Pacific

Journal of

Mathematics

PROXIMITY IN THE CURVE COMPLEX: BOUNDARY REDUCTION AND BICOMPRESSIBLE SURFACES

MARTIN SCHARLEMANN

Volume $228 \quad$ No. 2

December 2006 


\title{
PROXIMITY IN THE CURVE COMPLEX: BOUNDARY REDUCTION AND BICOMPRESSIBLE SURFACES
}

\author{
MARTIN SCHARLEMANN
}

\begin{abstract}
Suppose $N$ is a compressible boundary component of a compact irreducible orientable 3-manifold $M$, and $(Q, \partial Q) \subset(M, \partial M)$ is an orientable properly embedded essential surface in $M$, some essential component of which is incident to $N$ and no component is a disk. Let $\mathscr{V}$ and 2 denote respectively the sets of vertices in the curve complex for $N$ represented by boundaries of compressing disks and by boundary components of $Q$. We prove that, if $Q$ is essential in $M$, then $d(\mathscr{V}, 2) \leq 1-\chi(Q)$.

Hartshorn showed that an incompressible surface in a closed 3-manifold puts a limit on the distance of any Heegaard splitting. An augmented version of our result leads to a version of Hartshorn's theorem for merely compact 3-manifolds.

Our main result is: If a properly embedded connected surface $Q$ is incident to $N$, and $Q$ is separating and compresses on both its sides, but not by way of disjoint disks, then either $d(\mathscr{V}, 2) \leq 1-\chi(Q)$, or $Q$ is obtained from two nested connected incompressible boundary-parallel surfaces by a vertical tubing.

Forthcoming work with M. Tomova will show how an augmented version of this theorem leads to the same conclusion as Hartshorn's theorem, not from an essential surface, but from an alternate Heegaard surface. That is, if $Q$ is a Heegaard splitting of a compact $M$ then no other Heegaard splitting has distance greater than twice the genus of $Q$.
\end{abstract}

\section{Introduction}

Suppose $N$ is a compressible boundary component of an orientable irreducible 3-manifold $M$ and $(Q, \partial Q) \subset(M, \partial M)$ is an essential orientable surface in $M$, an essential component of which is incident to $N$ and no component of $Q$ is a disk. Let $\mathscr{V}$ and 2 denote sets of vertices in the curve complex for $N$ represented, respectively, by boundaries of compressing disks and by boundary components of $Q$. We will show:

MSC2000: primary 57N10; secondary 57M50.

Keywords: Heegaard splitting, strongly irreducible, handlebody, weakly incompressible.

Research partially supported by an NSF grant. 
Theorem. The distance $d(\mathscr{V}, 2)$ in the curve complex of $N$ is no greater than $1-\chi(Q)$. Furthermore, if no component of $Q$ is an annulus $\partial$-parallel into $N$, then, for each component $q$ of $Q \cap N$, we have $d(q, \mathscr{V}) \leq 1-\chi(Q)$.

A direct consequence is this generalization of a theorem of Hartshorn [2002]:

Theorem. If $P$ is a Heegaard-splitting surface for a compact orientable manifold $M$, and $(Q, \partial Q) \subset(M, \partial M)$ is a properly embedded incompressible surface, then $d(P) \leq 2-\chi(Q)$.

Both results are unsurprising, and perhaps well known (see, for example, [Bachman and Schleimer 2005] for a discussion of this in the broader setting of knots in bridge position with respect to a Heegaard surface).

It would be of interest to be able to prove the second result (Hartshorn's theorem) when $Q$ is a Heegaard surface, rather than an incompressible surface. Of course this is hopeless in general: a second copy of $P$ could be used for $Q$, and that would in general provide no information at all about the distance of the splitting $P$. However, suppose it is stipulated that $Q$ is not isotopic to $P$. One possibility is that $Q$ is weakly reducible. In that case (see [Casson and Gordon 1987]), it is either the stabilization of a lower-genus Heegaard splitting (to which we revert) or it gives rise to a lower-genus incompressible surface, and this allows the direct application of Hartshorn's theorem. So, in trying to extend Hartshorn's theorem to when $Q$ is a Heegaard surface, it suffices to consider the case in which $Q$ is strongly irreducible.

Here we carry out the first step in the extension of Hartshorn's theorem to the case in which $Q$ is a Heegaard surface. This first step is much like the first theorem quoted above. Specifically, we establish that bicompressible but weakly incompressible surfaces typically do not have boundaries that are distant in the curve complex from curves that compress in $M$.

Theorem. Suppose a properly embedded surface $Q$ is connected, separating, and incident to $N$. If $Q$ compresses on both its sides, but not by way of disjoint disks, then either:

- $d(\mathscr{V}, 2) \leq 1-\chi(Q)$; or

- $Q$ is obtained from two nested connected boundary-parallel surfaces by a vertical tubing.

Using this result, forthcoming work will demonstrate, via a two-parameter argument much as in [Rubinstein and Scharlemann 1996], that the genus of an alternate Heegaard splitting $Q$ does indeed establish a bound on the distance of $P$.

Maggy Tomova has provided valuable input to this proof. Beyond sharpening the foundational proposition (Propositions 2.5 and Theorem 5.4) in a very useful way, she provided an improved proof of Theorem 3.1. 


\section{Preliminaries and first steps}

First, we recall some definitions and elementary results, most of which are well known.

Definition 2.1. A $\partial$-compressing disk for $Q$ is a disk $D \subset M$ so that $\partial D$ is the end-point union of two arcs, $\alpha=D \cap \partial M$ and $\beta=D \cap Q$, and $\beta$ is essential in $Q$.

Definition 2.2. A surface $(Q, \partial Q) \subset(M, \partial M)$ is essential if it is incompressible and has a component that is not boundary-parallel. An essential surface is strictly essential if it has at most one non-annulus component.

Lemma 2.3. Suppose $(Q, \partial Q) \subset(M, \partial M)$ is a properly embedded surface and $Q^{\prime}$ is the result of $\partial$-compressing $Q$.

(1) If $Q$ is incompressible, so is $Q^{\prime}$.

(2) If $Q$ is essential, so is $Q^{\prime}$.

Proof. A description, dual to the boundary-compression from $Q$ to $Q^{\prime}$, is this: $Q$ is obtained from $Q^{\prime}$ by tunneling along an arc $\gamma$ dual to the $\partial$-compression disk. (The precise definition of tunneling is given in Section 4.) Certainly, any compressing disk for $Q^{\prime}$ in $M$ is unaffected by this operation near the boundary. Since $Q$ is incompressible, so is $Q^{\prime}$. This proves the first claim.

Suppose now that every component of $Q^{\prime}$ is boundary-parallel, and the arc $\gamma$ that is dual to the $\partial$-compression has ends on components $Q_{0}^{\prime}$ and $Q_{1}^{\prime}$ of $Q^{\prime}$ (possibly, $Q_{0}^{\prime}=Q_{1}^{\prime}$ ). If $\gamma$ is disjoint from the subsurfaces $P_{0}$ and $P_{1}$ of $\partial M$ to which $Q_{0}^{\prime}$ and $Q_{1}^{\prime}$, respectively, are parallel, then tunneling along $\gamma$ merely creates a component that is again boundary-parallel (to the band-sum of the $P_{i}$ along $\gamma$ ), thus contradicting the assumption that not all components of $Q$ are boundary-parallel. So suppose $\gamma$ lies in $P_{0}$, say. If both ends of $\gamma$ lie on $Q_{0}^{\prime}$ (so $Q_{1}^{\prime}=Q_{0}^{\prime}$ ), then the disk $\gamma \times I$ in the product region between $Q_{0}^{\prime}$ and $P_{0}$ would be a compressing disk for $Q$, which contradicts the incompressibility of $Q$.

Finally, suppose $Q_{1}^{\prime} \neq Q_{0}^{\prime}$, so $P_{0} \subset P_{1}$ and $\gamma$ is an arc in $P_{1}-P_{0}$ connecting $\partial P_{0}$ to $\partial P_{1}$. However, $P_{0}$ is not a disk, else the $\operatorname{arc} \beta$ in which the $\partial$-compressing disk intersects $Q$ would not have been essential in $Q$. So there is an essential simple closed curve $\gamma_{0} \subset P_{0}$ based at the point $\gamma \cap P_{0}$. Attach a band to $\gamma_{0}$ along $\gamma$ to get an $\operatorname{arc} \gamma_{+} \subset P_{1}$ with both ends on $\partial P_{1}$. Then the disk $E_{1}=\gamma_{+} \times I$, lying between $P_{1} \subset \partial M$ and $Q_{1}^{\prime}$, intersects $Q$ in a single arc, parallel in $M$ to $\gamma_{+}$and lying in the union of the top of the tunnel and $Q_{0}^{\prime}$. This arc divides $E_{1}$ into two disks; let $E$ be the one not incident to $\partial M$. Then $E$ has its boundary entirely in $Q$ and, since it is essential there, $E$ is a compressing disk for $Q-$ again a contradiction. See the figure on the next page. From these various contradictions we conclude that at least one of the components of $Q^{\prime}$ to which the ends of $\gamma$ are attached is not $\partial$-parallel, so $Q^{\prime}$ is essential. 

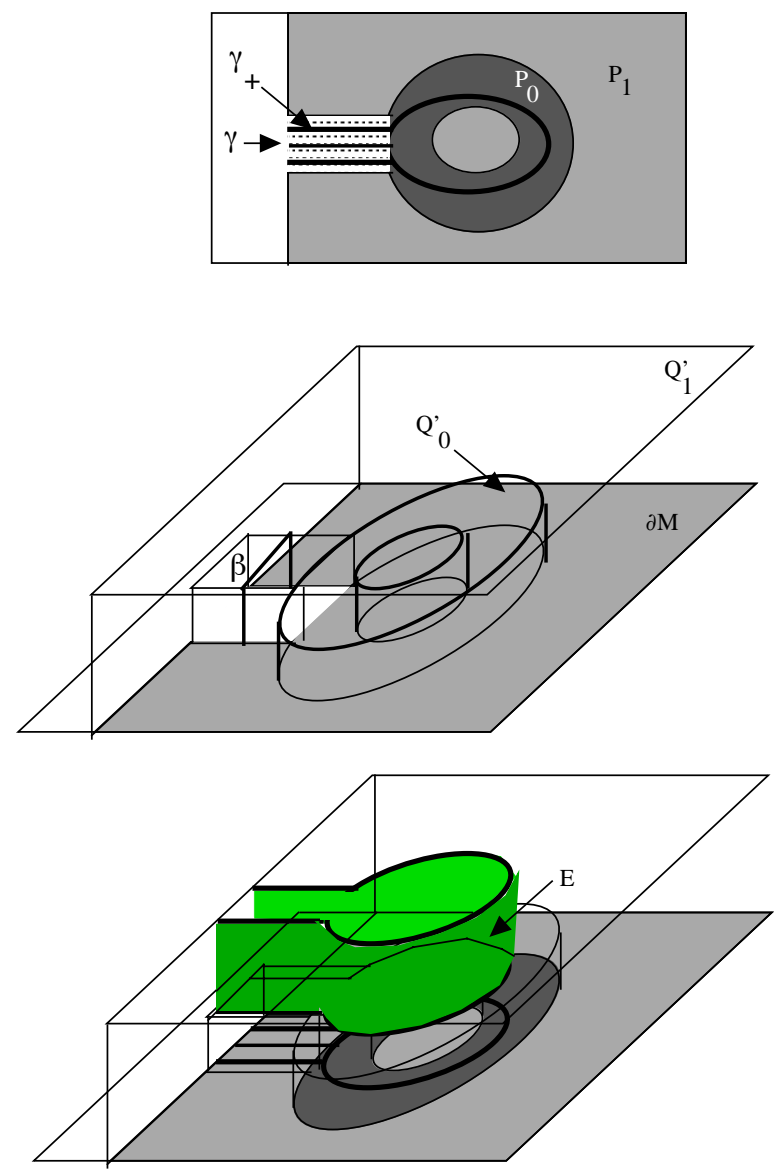

Definition 2.4. Suppose $S$ is a closed orientable surface, and $\alpha_{0}, \ldots, \alpha_{n}$ is a sequence of essential simple closed curves in $S$, so that for each $1 \leq i \leq n, \alpha_{i-1}$ and $\alpha_{i}$ can be isotoped to be disjoint. We say that the sequence is a length-n path in the curve complex of $S$ (see [Hempel 2001]).

The distance $d(\alpha, \beta)$ between a pair $\alpha, \beta$ of essential simple closed curves in $S$ is the smallest $n \in \mathbb{N}$ so that there is a path in the curve-complex from $\alpha$ to $\beta$ of length $n$. Curves are isotopic if and only if they have distance 0 .

Two sets of curves $\mathscr{V}, \mathscr{W}$ in $S$ have distance $d(\mathscr{V}, \mathscr{W})=n$ if $n$ is the smallest distance from a curve in $\mathscr{V}$ to a curve in $\mathscr{W}$.

Proposition 2.5. Suppose $M$ is an irreducible compact orientable 3-manifold, $N$ is a compressible component of $\partial M$, and $(Q, \partial Q) \subset(M, \partial M)$ is a properly embedded essential surface with $\chi(Q) \leq 1$ and at least one essential component incident to $N$. Let $\mathcal{V}$ be the set of essential curves in $N$ that bound disks in $M$, and let $q$ be any component of $\partial Q$. 
- If $Q$ contains an essential disk incident to $N$, then $d(\mathscr{V}, q) \leq 1$.

- If $Q$ does not contain any disk components, then $d(\mathscr{V}, q) \leq 1-\chi(Q)$, or $Q$ is strictly essential and $q$ lies in the boundary of a $\partial$-parallel annulus component of $Q$.

Proof. If $Q$ contains an essential disk $D$ incident to $N$, then $\partial D \in \mathscr{V}$. The component $q$ may be $\partial D$, or it may be another component of $\partial Q$, but in either case $d(\mathscr{V}, q) \leq 1$.

Suppose $Q$ contains no disks at all, and thus $\chi(Q) \leq 0$. Let $E$ be a compressing disk for $N$ in $M$ so that $|E \cap Q|$ is minimal among all such disks. Circles of intersection between $Q$ and $E$ and arcs of intersection that are inessential in $Q$ can be removed by isotoping $E$ via standard innermost-disk and outermost-arc arguments, so this choice of $E$ guarantees that $E$ and $Q$ only intersect along arcs that are essential in $Q$. If in fact they don't intersect at all, then $d(\partial E, q) \leq 1$ for every $q \in \partial Q$, and we are done. Consider, then, an $\operatorname{arc} \beta$ of $Q \cap E$ that is outermost in $E$, cutting off from $E$ a $\partial$-compressing disk $E_{0}$ for $Q$ that is incident to $N$. Boundary compressing $Q$ along $E_{0}$ gives (by Lemma 2.3) a new essential surface $Q^{\prime} \subset M$ that can be isotoped so that each component of $\partial Q^{\prime}$ is disjoint from each component of $\partial Q$. That is, for each component $q$ of $\partial Q$ and each component $q^{\prime}$ of $\partial Q^{\prime}$ we have that $d\left(q, q^{\prime}\right) \leq 1$.

The proof now is by induction on $1-\chi(Q)$. As $Q$ has no disk components, $1-\chi(Q) \geq 1$. Suppose $1-\chi(Q)=1$, that is, all components of $Q$ are annuli, so $Q$ is strictly essential. As we are not making any claims about the curves in 2 coming from d-parallel annuli components, we may assume all annuli in $Q$ are essential. Then $Q^{\prime}$ contains a compressing disk $D$ for $N$ (the result of boundary-reducing an essential annulus component of $Q$ along $E_{0}$ ), and $\partial D$ is disjoint from all $q \in \partial Q$. As $\partial D \in \mathscr{V}$,

as desired.

$$
d(q, \mathscr{V}) \leq 1=1-\chi(Q)
$$

Now suppose that $1-\chi(Q)>1$. If $Q$ is not strictly essential, then it contains at least two non-annulus components and, since it is essential, at least one essential component. Thus, there is a component $Q_{0}$ of $Q$ that is essential and such that $1-\chi\left(Q_{0}\right)<1-\chi(Q)$. By the induction hypothesis, for each component $q_{0}$ of $\partial Q_{0}$, we have $d\left(q_{0}, \mathscr{V}\right) \leq 1-\chi\left(Q_{0}\right)$. Of course, $d\left(q, q_{0}\right) \leq 1$ as well. Combining these inequalities, we obtain the desired result.

Suppose next that $Q$ is strictly essential, and again all $\partial$-parallel annuli have been removed prior to the boundary-compression described above. If the boundarycompression creates a disk component of $Q^{\prime}$, then it must be essential and incident to $N$, so $\partial D \in \mathscr{V}$ and, for every $q \in \partial Q$,

$$
d(q, \mathscr{V}) \leq d(q, \partial D) \leq 1 \leq 1-\chi(Q)
$$


and we are done. Suppose then that no component of $Q^{\prime}$ is a disk, and $q_{1}$ is any boundary component of an essential component $Q_{1}$ of $Q^{\prime}$. As

$$
1-\chi\left(Q_{1}\right) \leq 1-\chi\left(Q^{\prime}\right)<1-\chi(Q),
$$

the induction hypothesis applies, and

$$
d\left(q_{1}, \mathscr{V}\right) \leq 1-\chi\left(Q_{1}\right)<1-\chi(Q) .
$$

Since, for every component $q$ of $\partial Q$, we have $d\left(q, q_{1}\right) \leq 1$, the inequality

$$
d(q, \mathscr{V}) \leq d\left(q_{1}, \mathscr{V}\right)+d\left(q, q_{1}\right) \leq 1-\chi\left(Q^{\prime}\right)+1=1-\chi(Q)
$$

follows, as desired.

In order to prove Hartshorn's theorem on Heegaard splittings, it will be helpful to understand what it takes to be an essential surface in a compression body. Recall:

Definition 2.6 [Scharlemann 2002]. A compression body $H$ is a connected 3manifold obtained from a closed surface $\partial_{-} H$ by attaching 1-handles to $\partial_{-} H \times$ $\{1\} \subset \partial_{-} H \times I$. (It is conventional to consider a handlebody to be a compression body in which $\partial_{-} H=\varnothing$.) Dually, $H$ is obtained from a connected surface $\partial_{+} H$ by attaching 2-handles to $\partial_{+} H \times\{1\} \subset \partial_{+} H \times I$ and 3-handles to any 2-spheres thereby created. The cores of the 2-handles are called meridian disks, and a collection of meridian disks is called complete if its complement is $\partial_{-} H \times I$, together perhaps with some 3-balls.

Suppose two compression bodies $H_{1}$ and $H_{2}$ have $\partial_{+} H_{1} \simeq \partial_{+} H_{2}$. Glue $H_{1}$ and $H_{2}$ together along $\partial_{+} H_{i}=S$. The resulting compact 3-manifold $M$ can be written $M=H_{1} \cup_{S} H_{2}$, and this structure is called a Heegaard splitting of the 3-manifold with boundary $M$ (or, more specifically, of the triple $\left(M ; \partial_{-} H_{1}, \partial_{-} H_{2}\right)$ ). It is easy to show that every compact 3-manifold has a Heegaard splitting.

The following is probably well-known:

Lemma 2.7. Suppose $H$ is a compression body, and $(Q, \partial Q) \subset(H, \partial H)$ is incompressible. If $\partial Q \cap \partial_{+} H=\varnothing$, then $Q$ is inessential; that is, each component is d-parallel.

Proof. It suffices to consider the case in which $Q$ is connected. To begin with, consider the degenerate case in which $H=\partial_{-} H \times I$. Suppose there is a counterexample; let $Q$ be a counterexample that maximizes $\chi(Q)$.

Case 1: $H=\partial_{-} H \times I$ and $Q$ has nonempty boundary. $Q$ cannot be a disk, since $\partial_{-} H \times I$ is $\partial$-irreducible, so $\chi(Q) \leq 0$. By hypothesis, $\partial Q \subset \partial_{-} H \times\{0\}$. Choose $\alpha \subset \partial_{-} H \times\{0\}$ to be any curve that cannot be isotoped off of $\partial Q$, and let $A=\alpha \times I$ be the corresponding annulus in $\partial_{-} H \times I$. Minimize by isotopy of $A$ the number of components of $Q \cap A$. A standard argument shows that there are no inessential 
circles of intersection, and that each arc of intersection is essential in $Q$. Since $\partial Q$ is disjoint from $\partial_{-} H \times\{1\}$, all arcs of $Q \cap A$ have both ends in $\partial_{-} H \times\{0\}$. An outermost such arc in $A$ defines a $\partial$-compression of $Q$. The resulting surface $Q^{\prime}$ is still incompressible (since a compressing disk for $Q^{\prime}$ would persist into $Q$ ), and has at most two components, each of higher Euler characteristic; thus, each is $\partial$-parallel into $\partial_{-} H$. If there are two components, neither is a disk, or else the arc along which $\partial$-compression was supposedly performed would not have been essential. If there are two components of $Q^{\prime}$ and they are not nested (that is, each is parallel to the boundary in the complement of the other), it follows that $Q$ was $\partial$-parallel. If $Q^{\prime}$ had two nested components, it would follow that $Q$ was compressible, a contradiction. (See the end of the proof of Lemma 2.3, or the figure on page 328.) Similarly, if $Q^{\prime}$ is connected, then - depending on whether the tunneling arc dual to the $\partial$-compression lies inside or outside the region of parallelism between $Q^{\prime}$ and $\partial M-Q$ would either be compressible or itself $\partial$-parallel.

Case 2: $H=\partial_{-} H \times I$ and $Q$ is closed. Let

$$
A=\alpha \times I \subset \partial_{-} H \times I
$$

be any incompressible spanning annulus. A simple homology argument shows that $Q$ intersects $A$. After the standard move eliminating innermost disks, all intersection components are essential curves in $A$. Let $\lambda$ be the curve that is closest to $\partial_{-} H \times\{0\}$ in $A$. Let $Q^{\prime}$ be the properly embedded surface (now with boundary) obtained from $Q$ by removing a neighborhood of $\lambda$ in $Q$ and attaching two copies of the subannulus of $A$ between $\alpha \times\{0\}$ and $\lambda$. It's easy to see that $Q^{\prime}$ is still incompressible and its boundary is still disjoint from $\partial_{-} H \times\{1\}$, and that now $Q^{\prime}$ has nonempty boundary, so, by Case $1, Q^{\prime}$ is $\partial$-parallel. The subsurface of $\partial M$ to which $Q^{\prime}$ is $\partial$-parallel can't contain the neighborhood $\eta$ of $\alpha \times\{0\}$ in $\partial M$, or else the parallelism would identify a compressing disk for $Q$. It follows that the parallelism is outside of $\eta$, and so can be extended across $\eta$ to give a parallelism between $Q$ and a subsurface (hence a collection of components) of $\partial_{-} H \times\{0\}$.

Case 3: General case. Let $\Delta$ be a complete family of meridian disks for $H$, so that, when $H$ is compressed along $\Delta$, it becomes a product $\partial_{-} H \times I$. Since $Q$ is incompressible, a standard innermost-disk argument allows $\Delta$ to be redefined so that $\Delta \cap Q$ has no simple closed curves of intersection. Since $Q \cap \partial_{+} H=\varnothing$, it follows that $Q \cap \Delta=\varnothing$. Then, in fact, $Q \subset \partial_{-} H \times I$, and the result is deduced from Cases 1 or 2 .

\section{Hartshorn's theorem}

Using Proposition 2.5, we give a quick proof of Hartshorn's theorem (actually, of an extension to the case in which $M$ is not closed). Recall that the distance $d(P)$ of 
a Heegaard splitting [Hempel 2001] is the minimum distance in the curve complex of $P$ between a vertex representing a meridian curve on one side of $P$ and a vertex representing a meridian curve on the other side.

Theorem 3.1. If $P$ is a Heegaard splitting surface for a compact orientable manifold $M$, and $(Q, \partial Q) \subset(M, \partial M)$ is a connected essential surface, then $d(P) \leq$ $2-\chi(Q)$.

Remark that, as long as $Q$ contains no inessential disks or spheres and at most one essential disk or sphere, $Q$ need not be connected.

Proof. The next facts about Heegaard splittings are classical (see [Scharlemann 2002]): If $Q$ is a sphere, then $P$ is reducible, and hence $d(P)=0$. If $Q$ is a disk, then $P$ is $\partial$-reducible, so $d(P) \leq 1$. If neither occurs, then $M$ is irreducible and $\partial$-irreducible, which is what we henceforth assume. Moreover, once $Q$ is neither a disk nor a sphere, we have $2-\chi(Q) \geq 2$, so we might as well assume $d(P) \geq 2$, that is, $P$ is strongly irreducible.

Let $A$ and $B$ be the compression-bodies into which $P$ divides $M$, and let $\Sigma^{A}, \Sigma^{B}$ be spines of $A$ and $B$ respectively; that is, $\Sigma^{A}$ is the union of a graph in $A$ with $\partial_{-} A$, and $\Sigma^{B}$ is the union of a graph in $B$ with $\partial_{-} B$, so that $M-\left(\Sigma^{A} \cup \Sigma^{B}\right)$ is homeomorphic to $P \times(-1,1)$. We consider the curves $P \cap Q$ as $P$ sweeps from a neighborhood of $\Sigma^{A}$ (that is, near $P \times\{-1\}$ ) to a neighborhood of $\Sigma^{B}$ (near $P \times\{1\})$. Under this parameterization, let $P_{t}$ denote $P \times\{t\}$.

If $Q \cap \Sigma^{A}=\varnothing$, then $Q$ is an incompressible surface in the compression body Closure $\left(Q-\Sigma^{A}\right) \cong B$. By Lemma 2.7, $Q$ would be inessential, so this case does not arise. Similarly, we conclude that $Q$ must intersect $\Sigma^{B}$. It follows that, when $t$ is near $-1, P_{t} \cap Q$ contains meridian circles for $A$; when $t$ is near 1 , it contains meridian circles for $B$. Since $P$ is strongly irreducible, it can never be the case that both occur, so at some generic level neither will occur (see [Scharlemann 2002] for details, including why we can take such a level to be generic). Hence, there is a generic $t_{0}$ so that $P_{t_{0}} \cap Q$ contains no meridian circles for $P$.

An innermost inessential circle of intersection in $P_{t_{0}}$ must be inessential in $Q$ since $Q$ is incompressible. So all such circles of intersection can be removed by an isotopy of $Q$. After this process, all remaining curves of intersection are essential in $P_{t_{0}}$. Since $P_{t_{0}} \cap Q$ contains no meridian circles for $P$, no remaining circle of intersection can be inessential in $Q$ either. Hence, all components of $P_{t_{0}} \cap Q$ are essential in both surfaces; in particular, no component of $Q-P_{t_{0}}$ is a disk. At this point, revert to $P$ as notation for $P_{t_{0}}$.

If $P \cap Q=\varnothing$, then we are done, just as in the case in which $Q$ is disjoint from a spine. Similarly, we are done if the surface $Q_{A}=Q \cap A$ is inessential (and hence $\partial$-parallel) in $A$, or if $Q_{B}=Q \cap B$ is inessential in $B$. We conclude that $Q_{A}$ 
and $Q_{B}$ are both essential in $A$ and $B$, respectively, and the positioning of $P$ has guaranteed that no component of either is a disk.

Unless $Q_{A}$ and $Q_{B}$ are both strictly essential, the proof follows easily from Proposition 2.5: Suppose, for example, that $Q_{A}$ is not strictly essential, and let $U$ and $\mathscr{V}$ be the set of curves in $P$ bounding disks in $A$ and $B$, respectively. Let $q$ be a curve in $P \cap Q$ lying on the boundary of an essential component of $Q_{B}$. Then Proposition 2.5 says that $d(q, \mathcal{U}) \leq 1-\chi\left(Q_{A}\right)$ and $d(q, \mathscr{V}) \leq 1-\chi\left(Q_{B}\right)$, so

$$
\begin{aligned}
d(P) & =d(U, \mathscr{V}) \leq d(q, \mathscr{U})+d(q, \mathscr{V}) \leq\left(1-\chi\left(Q_{A}\right)\right)+\left(1-\chi\left(Q_{B}\right)\right) \\
& =2-\chi(Q),
\end{aligned}
$$

as required.

The case in which $Q_{A}$ and $Q_{B}$ are strictly essential is only a bit more difficult: Imagine coloring in red or blue each component of $Q_{A}$ or $Q_{B}$, respectively, that is not a $\partial$-parallel annulus. Since $Q_{A}$ and $Q_{B}$ are both essential, there are red and blue regions in $Q-P$. As $Q$ is connected, there is a path in $Q$ (possibly of length 0 ) with one end at a red region, one end at a blue region, and no interior point in a colored region. Since the interior of the entire path lies in a collection of $\partial$-parallel annuli, it follows that the curves in $P \cap Q$ to which the ends of the path are incident are isotopic curves in $P$. Now, apply the previous argument to a curve $q \subset P$ in that isotopy class of curves in $P$.

\section{Sobering examples of large distance}

It is natural to ask whether Proposition 2.5 can, in any useful way, be extended to surfaces that are not essential. It appears unlikely. If one allows $Q$ to be $\partial$-parallel, obvious counterexamples are easy to find: take a simple closed curve $\gamma$ in $N$ that is arbitrarily distant from $\mathcal{V}$, and use for $Q$ a $\partial$-parallel annulus $A$ constructed by pushing a regular neighborhood of $\gamma$ slightly into $M$. Even if one rules out $\partial-$ parallel surfaces but does allow $Q$ to be compressible, a counterexample is obtained by tubing, say, a possibly knotted torus in $M$ to an annulus $A$ as just constructed.

On the other hand, it has been a recent theme in the study of embedded surfaces in 3-manifolds that, for many purposes, a connected separating surface $Q$ in $M$ will behave much like an incompressible surface if $Q$ compresses to both sides, but not via disjoint disks. Would such a condition on $Q$ be sufficient to guarantee the conclusion of Proposition 2.5? That is:

Question 4.1. Suppose $M$ is an irreducible compact orientable 3-manifold, and $N$ is a compressible boundary component of $M$. Let $\mathscr{V}$ be the set of essential curves in $N$ that bound disks in $N$. Suppose further that $(Q, \partial Q) \subset(M, \partial M)$ is a connected separating surface, and $q$ is any boundary component of $Q$. If $Q$ is compressible 
into both complementary components, but not via disjoint disks, must it be true that $d(q, \mathscr{V}) \leq 1-\chi(Q)$ ?

In this section we show that there is an example for which the answer to Question 4.1 is "no". More remarkably, the next section will show that it is the only type of bad example.

A bit of terminology is useful. Regard $\partial D^{2}$ as the end-point union of two arcs, $\partial_{+} D^{2}$ and $\partial_{-} D^{2}$.

Definition 4.2. Suppose that $Q \subset M$ is a properly embedded surface, and $\gamma \subset$ Interior $(M)$ is an embedded arc incident to $Q$ precisely at $\partial \gamma$. There is a relative tubular neighborhood $\eta(\gamma) \cong \gamma \times D^{2}$ so that $\eta(\gamma)$ intersects $Q$ exactly in the two disk-fibers at the ends of $\gamma$. The surface obtained from $Q$ by removing these two disks and attaching the cylinder $\gamma \times \partial D^{2}$ is said to be obtained by tubing along $\gamma$.

Definition 4.3. Similarly, suppose that $\gamma \subset \partial M$ is an embedded arc incident to $\partial Q$ precisely in $\partial \gamma$. There is a relative tubular neighborhood $\eta(\gamma) \cong \gamma \times D^{2}$ so that $\eta(\gamma)$ intersects $Q$ precisely in the two $D^{2}$ fibers at the ends of $\gamma$ and $\eta(\gamma)$ intersects $\partial M$ exactly in the rectangle $\gamma \times \partial_{-} D^{2}$. The properly embedded surface obtained from $Q$ by removing the two $D^{2}$-fibers at the ends of $\gamma$ and attaching the rectangle $\gamma \times \partial_{+} D^{2}$ is said to be obtained by tunneling along $\gamma$.

Let $P_{0}$ and $P_{1}$ be two connected compact subsurfaces in the same component $N$ of $\partial M$, with each component of $\partial P_{0}$ and $\partial P_{1}$ essential in $\partial M$ and $P_{0} \subset \operatorname{Interior}\left(P_{1}\right)$. Let $Q_{1}$ be the properly embedded surface in $M$ obtained by pushing $P_{1}$ rel $\partial$ into the interior of $M$. Let $Q_{0}$ denote the properly embedded surface obtained by pushing $P_{0}$ rel $\partial$ into the collar between $P_{1}$ and $Q_{1}$. The region $R$ lying between $Q_{0}$ and $Q_{1}$ is naturally homeomorphic to $Q_{1} \times I$. (Here, $\partial Q_{1} \times I$ can be thought of either as vertically crushed to $\partial Q_{1} \subset \partial M$, or as constituting a small collar of $\partial Q_{1}$ in $P_{1} \subset \partial M$.) Under the homeomorphism $R \cong Q_{1} \times I$, the top of $R$ (corresponding to $Q_{1} \times\{1\}$ ) is $Q_{1}$, and the bottom of $R$ (corresponding to $Q_{1} \times\{0\}$ ) is the boundaryunion of $Q_{0}$ and $P_{1}-P_{0}$. The properly embedded surface $Q_{0} \cup Q_{1} \subset M$ is called the recessed collar determined by $P_{0} \subset P_{1}$ bounding $R$.

Recessed collars behave predictably under tunnelings:

Lemma 4.4. Suppose $Q_{0} \cup Q_{1} \subset M$ is the recessed collar determined by $P_{0} \subset$ Interior $\left(P_{1}\right)$, and $R \cong Q_{1} \times I$ is the component of $M-\left(Q_{0} \cup Q_{1}\right)$ on whose boundary both $Q_{0}$ and $Q_{1}$ lie. Let $\gamma \subset \partial M$ be a properly embedded arc in $\partial M-\left(Q_{0} \cup Q_{1}\right)$, and $Q_{+}$the surface obtained from $Q_{0} \cup Q_{1}$ by tunneling along $\gamma$.

(1) If $\gamma \subset P_{1}-P_{0}$ and $\gamma$ has both ends on $\partial P_{0}$, or if $\gamma \subset\left(\partial M-P_{1}\right)$, then $Q_{+}$is a recessed collar.

(2) If $\gamma \subset P_{0}$, then there is a compressing disk for $Q_{+}$in $M-R$. 
(3) If $\gamma \subset P_{1}-P_{0}$ and $\gamma$ has one or both ends on $\partial P_{1}$, then there is a compressing disk for $Q_{+}$in $R$.

Proof. In the first case, tunneling is equivalent to just adding a band to either $P_{1}$ or $P_{0}$, and then constructing the recessed collar. In the second case, the disk $\gamma \times I$ in the collar between $P_{0}$ and $Q_{0}$ determines a compressing disk for $Q_{+}$(that is, for the component of $Q_{+}$coming from $Q_{0}$ ) that lies outside $R$.

Similarly, in one of the third cases, when $\gamma \subset P_{1}-P_{0}$ has both ends on $\partial P_{1}$, $\gamma \times I$ in the collar between $P_{1}$ and $Q_{1}$ determines a compressing disk for $Q_{+}$(this time, for the component of $Q_{+}$coming from $Q_{1}$ ) that now lies inside $R$.

In the last case, when one end of $\gamma \subset P_{1}-P_{0}$ lies on each of $\partial P_{0}$ and $\partial P_{1}$, a slightly more sophisticated construction is needed. After the tunneling construction, $\partial Q_{+} \cap \operatorname{Interior}\left(P_{1}\right)$ has one arc-component $\gamma^{\prime}$, consisting of two parallel copies of the spanning arc $\gamma$ and a subarc of the component of $\partial P_{0}$ that is incident to $\gamma$. This arc, $\gamma^{\prime} \subset \partial Q_{+}$, can be pushed slightly into $Q_{+}$. Then the disk $\gamma^{\prime} \times I$ (using the product structure on $R$ ) determines a compressing disk for $Q_{+}$that lies in $R$. (The disk $\gamma^{\prime} \times I$ looks much like the disk $E$ in the figure from page 328.)

One of the constructions of Lemma 4.4 will be needed in a different context:

Lemma 4.5. Suppose $Q_{0} \cup Q_{1} \subset M$ and $Q_{1} \cup Q_{2} \subset M$ are the recessed collars determined by connected surfaces $P_{0} \subset \operatorname{Interior}\left(P_{1}\right)$ and $P_{1} \subset \operatorname{Interior}\left(P_{2}\right)$. Let $R_{1}$ and $R_{2}$ be the regions bounded by these recessed collars. Furthermore, let $\gamma_{1}, \gamma_{2} \subset \partial M$ be properly embedded arcs spanning $P_{1}-P_{0}$ and $P_{2}-P_{1}$, respectively; that is, $\gamma_{i}$ has one end-point on each of $\partial P_{i}$ and $\partial P_{i-1}$. If $Q_{+}$is the connected surface obtained from $Q_{0} \cup Q_{1} \cup Q_{2}$ by tunneling along both $\gamma_{1}$ and $\gamma_{2}$, then either

(1) there are disjoint compressing disks for $Q_{+}$in $R_{1}$ and $R_{2}$; or

(2) $P_{0}$ is an annulus parallel in $P_{1}$ to a component $c$ of $\partial P_{1}$, and $c$ is incident to both tunnels.

In the latter case, $Q_{+}$is properly isotopic to the surface obtained from the recessed collar $Q_{1} \cup Q_{2}$ by tubing along an arc in Interior $(M)$ that is parallel to $\gamma_{2} \subset \partial M$.

Proof. For $P$ any surface with boundary, define an eyeglass graph in $P$ to be the union of an essential simple closed curve in the interior of $P$ and an embedded arc in the curve's complement, connecting the curve to $\partial P$.

Let $c_{1} \subset \partial P_{1}$ and $c_{0} \subset \partial P_{0}$ be the components to which the ends of $\gamma_{1}$ are incident. Let $c_{2}$ be the component of $\partial P_{1}$ (note: not of $\partial P_{2}$ ) to which the end of $\gamma_{2}$ is incident. (It is possible that $c_{1}=c_{2}$.) Let $\alpha$ be any essential simple closed curve in $P_{0}$, and choose an embedded arc in $P_{0}-\alpha$ connecting $\alpha$ to the end of $\gamma_{1}$ in $c_{0}$; the union of that arc, the closed curve $\alpha$, and the arc $\gamma_{1}$ is an eyeglass curve $e_{1}$ in $P_{1}$ which intersects $P_{1}-P_{0}$ in the arc $\gamma_{1}$. Then the construction of Lemma 4.4 (applied there to the eyeglass $\gamma_{1} \cup c_{0}$ ) shows here that a neighborhood of the 
product $e_{1} \times I \subset R_{1} \cong P_{1} \times I$ contains a compressing disk for $Q_{+}$that lies in $R_{1}$ and which intersects $Q_{1}$ in a neighborhood of $e_{1} \times\{1\}$.

Similarly, for $\beta$ any essential simple closed curve in $P_{1}$, and an embedded arc in $P_{1}-\beta$ connecting $\beta$ to the end of $\gamma_{2}$ in $c_{2}$, we get an eyeglass $e_{2} \subset P_{2}$ and a compressing disk for $Q_{+}$that lies in $R_{2}$ and whose boundary intersects $Q_{1}$ only within a neighborhood of $e_{2} \times\{1\}$. So, if we can find such eyeglasses in $P_{1}$ and $P_{2}$ that are disjoint, then we will have constructed the required disjoint compressing disks.

Suppose first that $P_{0}$ is not an annulus parallel to $c_{1}$. Then $P_{0}$ contains an essential simple closed curve $\alpha$ that is not parallel to $c_{1}$. Since $\alpha$ is not parallel to $c_{1}$, no component of the complement $P_{1}-e_{1}$ is a disk, so there is an essential simple closed curve $\beta$ in the component of $P_{1}-e_{1}$ that is incident to $c_{2}$. The same is true even if $P_{0}$ is an annulus parallel to $c_{1}$, as long as $c_{1} \neq c_{2}$. This proves the enumerated conclusions. See figure.

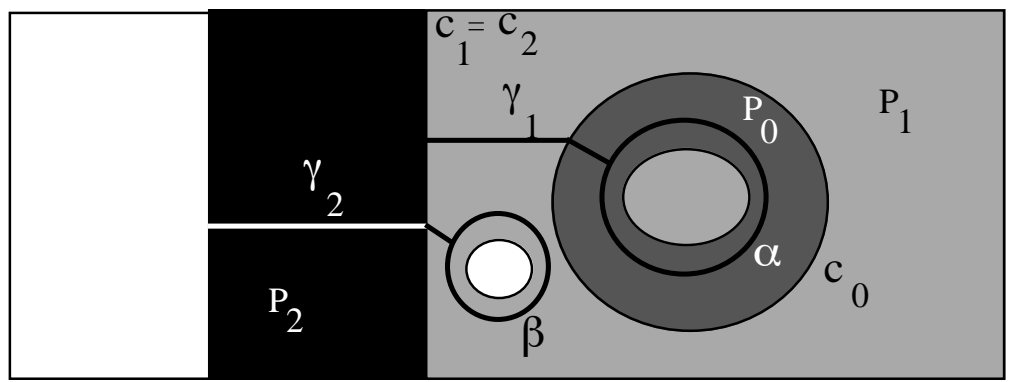

The proof that in case (2), $Q_{+}$can be described by tubing $Q_{1}$ to $Q_{2}$ along an arc parallel to $\gamma_{2}$ is a pleasant exercise left to the reader.

Consider now a particular type of tubing of a recessed collar. Suppose $Q_{0} \cup Q_{1} \subset$ $M$ is the recessed collar bounding $R$ determined by $P_{0} \subset P_{1} \subset \partial M$. Let $\rho$ denote a vertical spanning arc in $R$, that is, the image in $R \cong P_{1} \times I$ of point $\times I$, where point $\in P_{0}$. Let $Q$ be the surface obtained from $Q_{0} \cup Q_{1}$ by tubing along $\rho$. Then $Q$ is called a tube-spanned recessed collar.

A tube-spanned recessed collar has nice properties:

Lemma 4.6. If $Q$ is a tube-spanned recessed collar constructed as above, then:

(1) $Q$ is connected and separating, and $Q$ compresses in both complementary components in $M$.

(2) If $Q$ compresses in both complementary components via disjoint disks, then $P_{1} \subset \partial M$ is compressible in $M$.

(3) If $Q_{+}$is obtained from $Q$ by tunneling, then either $Q_{+}$is also a tube-spanned recessed collar, or $Q_{+}$compresses in both complementary components via disjoint disks. (Possibly both are true.) 
(4) If $Q_{+}$is obtained from $Q$ by tunneling together $Q$ and a $\partial$-parallel connected incompressible surface $Q^{\prime}$, then either $Q_{+}$is also a tube-spanned recessed collar, or $Q_{+}$compresses in both complementary components via disjoint disks. (Possibly both are true.)

Proof. The construction guarantees that $Q$ is connected and separating. It compresses on both sides: Let $Y$ denote the component $R-\eta(\rho)$ of $M-Q$, and let $X$ be the other component. A disk-fiber $\mu$ of $\eta(\rho)$ is a compressing disk for $Q$ in $X$. To see a compressing disk for $Q$ in $Y$, start with an essential simple closed curve in $Q_{0}$ containing the end of $\rho$ in $Q_{0}$. The corresponding vertical annulus $A \subset R$ includes the vertical arc $\rho \subset R$. Then $A-\eta(\rho)$ is a disk in $Y$ whose boundary is essential in $Q$.

To prove the second property, suppose that there are disjoint compressing disks, $D_{X} \subset X$ and $D_{Y} \subset Y$. The boundary $\partial D_{Y}$ cannot be disjoint from the meridian $\mu$ of $\eta(\rho)$, since if it were, $\partial D_{Y}$ would lie either in the top or the bottom of $Y \cong$ $\left(P_{1}-\right.$ point $) \times I$, either of which is clearly incompressible in $Y$. So $D_{X}$ cannot be parallel to $\mu$. A standard innermost-disk argument allows us to choose $D_{X}$ so that $D_{X} \cap \mu$ contains no circles of intersection, and an isotopy of $\partial D_{X}$ on $Q$ ensures that any arc component of $\partial D_{X}-\mu$ is essential in one of the punctured surfaces $Q_{1} \cap Q$ or $Q_{0} \cap Q$. If $D_{X}$ is disjoint from $\mu$, it lies on $Q_{1}$, say, but in any case it determines a compressing disk for $P_{1}$ in $M$, as required. If $D_{X}$ is not disjoint from $\mu$, then an outermost disk in $D_{X}$ cut off by $\mu$ would similarly determine a compression of $P_{1}$ in $M$.

The third property follows from Lemma 4.4. When the tunneling there leaves $Q_{+}$as a recessed collar (option 1), the operation here leaves $Q_{+}$a tube-spanned recessed collar. If the tunneling arc $\gamma$ lies in $P_{1}-P_{0}$ and thereby gives rise to a compressing disk in $R$ (option 3), the compressing disk $D_{Y}$ constructed there lies in $Y$, and so can clearly be kept disjoint from the vertical arc $\rho$. Then $D_{Y}$ is disjoint from the compressing disk $\mu$ for $X$, as required. Finally, if $\gamma$ lies in $P_{0}$ (option 2), the compressing disk $D_{X}$ in $M-R$ constructed there lies in $X$ and intersects $Q_{0}$ in a single essential arc. The simple closed curve in $Q_{0}$ from which $A$ is constructed can be taken to intersect $D_{X}$ in at most one point, so in the end the disk $D_{Y} \subset Y$ intersects $D_{X}$ in at most one point. Therefore, the boundary of a regular neighborhood of $\partial X \cup \partial Y$ in $Q$ is a simple closed curve that bounds a disk in both $X$ and $Y$, as required.

The fourth property is proved in a similar way. Suppose first that $\partial Q^{\prime}$ is disjoint from $P_{1}$. If the region $P^{\prime} \subset \partial M$ to which $Q^{\prime}$ is parallel is disjoint from $P_{1}$, then tunneling $Q^{\prime}$ to $Q_{1}$ just creates a larger $\partial$-parallel surface, and $Q_{+}$is a tube-spanned recessed collar. If $P_{1} \subset P^{\prime}$, the region $R^{\prime}$ between $Q^{\prime}$ and $Q_{1}$ is a recessed collar and, according to option 3 of Lemma 4.4, there is a compressing disk for $Q_{+}$in $R^{\prime} \cap X$ that is incident to $Q_{1}$ only in a collar of $\partial Q_{1}$. In particular, it is disjoint 
from a compressing disk for $Q$ in $R \cap Y$, constructed above from an annulus $A$ that is incident to $Q_{1}$ away from this collar.

Next, assume that $\partial Q^{\prime}$ lies in $P_{1}-P_{0}$, so that $P^{\prime} \subset P_{1}-P_{0}$. If the tunnel connects $Q^{\prime}$ to $Q_{0}$, then tunneling $Q_{0}$ to $Q^{\prime}$ just creates a larger $\partial$-parallel surface, and $Q_{+}$is a tube-spanned recessed collar. If the tunneling connects $Q^{\prime}$ to $Q_{1}$, the argument is the same as when $Q_{+}$is obtained from $Q$ by tunneling into $P_{1}-P_{0}$ with both ends of the tunnel on $\partial P_{1}$.

Finally, suppose that $\partial Q^{\prime}$ lies in $P_{0}$, so that $P^{\prime} \subset P_{0}$. Then the tunneling connects $Q^{\prime}$ to $Q_{0}$. The region $R^{\prime}$ between $Q^{\prime}$ and $Q_{0}$ is a recessed collar and, according to option 3 of Lemma 4.4, there is a compressing disk for $Q_{+}$in $R^{\prime} \cap X$ that is incident to $Q^{\prime}$ only in a collar of $\partial Q^{\prime}$. In particular, it is disjoint from the compressing disk for $Q$ in $R \cap Y$, constructed above from an annulus $A$ incident to $Q_{0}$ in the image of $P^{\prime} \subset P_{1}$ away from that collar.

Corollary 4.7. Suppose $M$ is an irreducible compact orientable 3-manifold, and $N$ is a compressible boundary component of $M$. Let $\mathscr{V}$ be the set of curves in $N$ that arise as boundaries of compressing disks of $N$. For any $n \in \mathbb{N}$, there is a connected properly imbedded separating surface $(Q, \partial Q) \subset(M, N)$ so that $Q$ compresses in both complementary components, but not via disjoint disks, and so that, for any component $q$ of $\partial Q, d(q, \mathscr{V}) \geq n$.

Proof. Let $A_{1}$ be an annulus in $\partial M$ whose core has distance at least $n$ from $\mathscr{V}$. Let $A_{0} \subset A_{1}$ be a thinner subannulus, and let $Q$ be the tube-spanned recessed product in $M$ that they determine. The result follows from the first two conclusions of Lemma 4.6.

\section{Any example is a tube-spanned recessed collar}

It will be useful to expand the context beyond connected separating surfaces.

Definition 5.1. Let $(Q, \partial Q) \subset(M, \partial M)$ be a properly embedded orientable surface in the orientable irreducible 3-manifold $M$. $Q$ will be called a splitting surface if no component is closed, no component is a disk, and $M$ is the union of two 3manifolds $X$ and $Y$ along $Q$.

We abbreviate by saying that $Q$ splits $M$ into the submanifolds $X$ and $Y$.

The definition differs slightly from [Jones and Scharlemann 2001, Definition 1.1], which allowed $Q$ to have closed components and disk components. Note also that the condition that $M$ be the union of two 3-manifolds $X$ and $Y$ along $Q$ is equivalent to saying that $Q$ can be normally oriented so that any oriented $\operatorname{arc}$ in $M$ transverse to $Q$ alternately crosses $Q$ in the direction consistent with the normal orientation and then against the normal orientation. 
Definition 5.2. Suppose, as above, that $(Q, \partial Q) \subset(M, \partial M)$ is a splitting surface that splits $M$ into submanifolds $X$ and $Y$. $Q$ is bicompressible if both $X$ and $Y$ contain compressing disks for $Q$ in $M$, and it is strongly compressible if there are such disks whose boundaries are disjoint in $Q$. If $Q$ is not strongly compressible then it is weakly incompressible.

Note that, if $Q$ is bicompressible but weakly incompressible, $\partial Q$ is necessarily essential in $\partial M$, for otherwise an innermost inessential component would bound a compressing disk for $Q$ in $Y \cap \partial M$, say. Such a disk, lying in $\partial M$, would necessarily be disjoint from any compressing disk for $Q$ in $X$.

There are natural extensions of these ideas. One that will eventually prove useful is the extension to $\partial$-compressions of splitting surfaces:

Definition 5.3. A splitting surface $(Q, \partial Q) \subset(M, \partial M)$ is strongly $\partial$-compressible if there are $\partial$-compressing disks $D_{X} \subset X$ and $D_{Y} \subset Y$ with $\partial D_{X} \cap \partial D_{Y}=\varnothing$.

Here is our main result:

Theorem 5.4. Suppose $M$ is an irreducible compact orientable 3-manifold, $N$ is a compressible boundary component of $M$, and $(Q, \partial Q) \subset(M, \partial M)$ is a bicompressible, weakly incompressible splitting surface, with a bicompressible component incident to $N$.

Let $\mathscr{V}$ be the set of essential curves in $N$ that bound disks in $M$. If $q$ is any component of $\partial Q \cap N$, then either

- $d(q, \mathscr{V}) \leq 1-\chi(Q)$ in the curve complex on $N$; or

- $q$ lies in the boundary of a $\partial$-parallel annulus component of $Q ;$ or

- the component of $Q$ containing $q$ is a tube-spanned recessed collar, and all other components incident to $N$ are incompressible and $\partial$-parallel.

Note that, in the last case, $Q$ lies entirely in a collar of $N$.

Lemma 5.5. Let $(Q, \partial Q) \subset(M, \partial M)$ and $N \subset \partial M$ be as in Theorem 5.4, so that $Q$ splits $M$ into $X$ and $Y$. If $Q_{X}$ is the result of maximally compressing $Q$ into $X$, then

(1) $Q_{X}$ is incompressible in $M$, and

(2) there is a compressing disk $D$ for $N$ in $M$ so that some complete set of compressing disks for $Q$ in $X$ is disjoint from $D$ and, moreover, $Q \cap D$ consists entirely of arcs that are essential in $Q_{X}$.

Proof. First we show that $Q_{X}$ is incompressible. This is, in a sense, a classical result, going back to Haken. A more modern view is in [Casson and Gordon 1987]. Here we take the viewpoint first used in [Scharlemann and Thompson 1994, Proposition 2.2], which adapts well to other contexts that we will need as well, and is a good source for details missing here. 
$Q_{X}$ is obtained from $Q$ by compressing into $X$. Dually, we can think of $Q_{X}$ as a surface splitting $M$ into $X^{\prime}$ and $Y^{\prime}$ (except that possibly $Q_{X}$ has some closed components) and $Q$ is constructed from $Q_{X}$ by tubing along a collection of arcs in $Y^{\prime}$. Sliding one of these arcs over another or along $Q_{X}$ merely moves $Q$ by an isotopy, so an alternate view of the construction is this: There is a graph $\Gamma \subset Y^{\prime}$, with all of its valence-one vertices on $Q_{X}$. A regular neighborhood of $Q_{X} \cup \Gamma$ has boundary consisting of a copy of $Q_{X}$ and a copy of $Q$. (This construction of $Q$ from $Q_{X}$ could be called 1-surgery along the graph $\Gamma$.) The graph $\Gamma$ may be varied by slides of edges along other edges or along $Q_{X}$; the effect on $Q$ is merely to isotope it in the complement of $Q_{X}$.

Suppose that $F$ is a compressing disk for $Q_{X}$ in $M$. Then $F$ must lie in $Y^{\prime}$, or else $Q$ could be further compressed into $X$. Choose a representation of $\Gamma$ which minimizes $|F \cap \Gamma|$, and then choose a compressing disk $E$ for $Q$ in $Y$ which minimizes $|F \cap E|$. If there are any closed components of $F \cap E$, an innermost one in $E$ bounds a subdisk of $E$ disjoint from $F, \Gamma$ and $Q$; an isotopy of $F$ will remove the intersection curve without raising $|F \cap \Gamma|$. So, in fact, there are no closed curves in $F \cap E$.

The disk $F$ must intersect the graph $\Gamma$, or else $F$ would lie entirely in $Y$ and so be a compressing disk for $Q$ in $Y$ that is disjoint from compressing disks of $Q$ in $X$. This would contradict the weak incompressibility of $Q$. One can view the intersection of $\Gamma \cup E$ with $F$ as a graph $\Lambda \subset F$ whose vertices are the points $\Gamma \cap F$ and whose edges are the $\operatorname{arcs} F \cap E$.

If there is an isolated vertex of the graph $\Lambda \subset F$ (that is, a point in $\Gamma \cap F$ that is disjoint from $E$ ), then the vertex would correspond to a compressing disk for $Q$ in $X$ that is disjoint from $E$, contradicting weak irreducibility. If there is a loop in $\Lambda \subset F$ whose interior contains no vertex, an innermost such loop would bound a subdisk of $F$ that could be used to simplify $E$; that means finding a compressing disk $E_{0}$ for $Q$ in $Y$ so that

$$
\left|F \cap E_{0}\right|<|F \cap E|
$$

again a contradiction. We conclude that $\Lambda$ has a vertex $w$ that is incident to edges but to no loops of $\Lambda$. Choose an $\operatorname{arc} \beta$ which is outermost in $E$ among all arcs of $F \cap E$ which are incident to $w$. Then $\beta$ cuts off from $E$ a disk $E^{\prime}$ with $E^{\prime}-\beta$ disjoint from $w$. Let $e$ be the edge of $\Gamma$ that contains $w$. The disk $E^{\prime}$ gives instructions about how to isotope and slide the edge $e$ until $w$, and possibly other points of $\Gamma \cap F$, is removed, lowering $|\Gamma \cap F|$, a contradiction that establishes the first claim.

To establish the second claim, first note that by shrinking very small a complete set of compressing disks for $Q$ in $X$, we can of course make them disjoint from 
any $D$; the difficulty is ensuring that $Q_{X} \cap D$ has no simple closed curves of intersection.

Choose $D$ and isotope $Q_{X}$ to minimize the number of components $\left|D \cap Q_{X}\right|$, then choose a representation of $\Gamma$ that minimizes $|D \cap \Gamma|$, and, finally, choose a compressing disk $E$ for $Q$ in $Y$ that minimizes $|D \cap E|$. If there are any closed components of $D \cap E$, an innermost one in $E$ bounds a subdisk of $E$ disjoint from $D, \Gamma$ and $Q$; an isotopy of $D$ will remove the intersection curve without raising either $\left|D \cap Q_{X}\right|$ or $|D \cap \Gamma|$. So, in fact, there are no closed curves in $D \cap E$.

Suppose there are closed curves in $D \cap Q_{X}$. An innermost one in $D$ will bound a subdisk $D_{0}$. Since $Q_{X}$ is incompressible, $\partial D_{0}$ also bounds a disk in $Q_{X}$; the curve of intersection could then be removed by an isotopy of $Q_{X}-$ a contradiction.

From this contradiction we deduce that all components of $D \cap Q_{X}$ are arcs. All arcs are essential in $Q_{X}$, or else $\left|D \cap Q_{X}\right|$ could be lowered by rechoosing $D$. The only other components of $D \cap Q$ are closed curves, compressible in $X$ and each corresponding to a point in $D \cap \Gamma$. So it suffices to show that $D \cap \Gamma=\varnothing$. The proof is analogous to the proof of the first claim, where it was shown that $\Gamma$ must be disjoint from any compressing disk $F$ for $Q_{X}$ in $Y^{\prime}$, but now, for $F$, we take a (disk) component of $D-Q_{X}$.

If no component of $D-Q_{X}$ intersects $\Gamma$, there is nothing to prove; so let $F$ be a component intersecting $\Gamma$, and regard

$$
\Lambda=(\Gamma \cup E) \cap F
$$

as a graph in $F$, with possibly some edges incident to the arcs $Q_{X} \cap D$ lying in $\partial F$. As above, no vertex of $\Lambda$ (that is, no point of $\Gamma \cap F$ ) can be isolated in $\Lambda$, and an innermost inessential loop in $\Lambda$ would allow an improvement in $E$ so as to reduce $D \cap E$. Hence, there is a vertex $w$ of $\Lambda$ that is incident to edges but not to loops in $\Lambda$. An edge in $\Lambda$ that, in $E$, is outermost among all edges incident to $w$ will cut off a disk from $E$ that provides instructions on how to slide the edge $e$ of $\Gamma$ containing $w$ so as to remove the intersection point $w$ and possibly other intersection points. As in the first case, some sliding of the end of $e$ may necessarily be along arcs in $Q_{X}$, as well as over other edges in $\Gamma$.

Proof of Theorem 5.4. Just as in the proof of Proposition 2.5, the argument is by induction on $1-\chi(Q)$. Since $Q$ contains no disk components, $1-\chi(Q) \geq 1$.

If compressing disks for $Q$ were incident to two different components of $Q$, then there would be compressing disks on opposite sides incident to two different components of $Q$, violating weak incompressibility. We deduce that all compressing disks for $Q$ are incident to at most one component $Q_{0}$ of $Q$. The component $Q_{0}$ cannot be an annulus, or else the boundaries of compressing disks in $X$ and $Y$ would be parallel in $Q_{0}$, and so could be made disjoint. If $Q$ also contains an 
essential component $Q^{\prime}$ incident to $N$, then

$$
1-\chi\left(Q^{\prime}\right) \leq 1-\chi\left(Q-Q_{0}\right)<1-\chi(Q),
$$

and so, by Proposition 2.5, for any component $q^{\prime}$ of $\partial Q^{\prime} \cap N$,

$$
d\left(q^{\prime}, \mathscr{V}\right) \leq 1-\chi\left(Q^{\prime}\right)<1-\chi(Q) .
$$

This implies that

$$
d(q, \mathscr{V}) \leq d\left(q^{\prime}, \mathscr{V}\right)+d\left(q, q^{\prime}\right) \leq 1-\chi(Q),
$$

as required. Thus, we will also henceforth assume that no component of $Q$ incident to $N$ is essential.

We can also assume that each component of $Q-Q_{0}$ is itself an incompressible surface. For assume $D$ is a compressing disk for a component $Q_{1} \neq Q_{0}$ of $Q$, chosen among all such disks to have a minimal number of intersection components with $Q$. If the interior of $D$ were disjoint from $Q$, then $D$ would be a compressing disk for $Q$ itself, violating weak incompressibility as described above. Similarly, an innermost circle of $Q \cap D$ in $D$ must lie in $Q_{0}$. Consider a subdisk $D^{\prime}$ of $D$ (possibly all of $D$ ) with the property that its boundary is second-innermost among components of $D \cap Q$. That is, the interior of $D^{\prime}$ intersects $Q$ exactly in innermost circles of intersection, each bounding disks in $X$, say. If $\partial D^{\prime}$ is not in $Q_{0}$, then it is also a compressing disk for $Q_{X}$, contradicting the first statement in Lemma 5.5. The argument is only a bit more subtle when $\partial D^{\prime}$ is in $Q_{0}$, see the No Nesting Lemma [Scharlemann 1998, Lemma 2.2].

Let $Q_{-}$be the union of components of $Q$ that are not incident to $N$. Since $Q_{-}$ is incompressible, each compressing disk for $N$ is disjoint from $Q_{-}$. In particular, it suffices to work inside the 3-manifold $M-\eta\left(Q_{-}\right)$instead of $M$. So, with no loss of generality, we can assume that $Q_{-}=\varnothing$, in other words, that each component of $Q$ is incident to $N$.

Since each component of $Q$ other than $Q_{0}$ is incompressible and not essential, each is boundary-parallel. In particular, removing one of these components $Q_{1}$ from $Q$ still leaves a bicompressible, weakly incompressible splitting surface, even though each component of $M-Q_{1}$ in the region of parallelism between $Q_{1}$ and $\partial M$ would need to be switched from $X$ to $Y$ or vice versa. Since we don't care about the boundaries of $\partial$-parallel annuli, all such components can be removed from $Q$ without affecting the hypotheses or conclusion. If there remains a $\partial$-parallel component $Q_{1}$ that is not an annulus, then consider $Q^{\prime}=Q-Q_{1}$. We have

$$
1-\chi\left(Q^{\prime}\right)<1-\chi(Q),
$$

so the inductive hypothesis applies. Then either $Q_{0}$ is a tube-spanned recessed 
collar (and we are done) or, for any component $q^{\prime}$ of $\partial Q^{\prime}$,

$$
d\left(q^{\prime}, \mathscr{V}\right) \leq 1-\chi\left(Q^{\prime}\right)<1-\chi(Q) .
$$

This implies that

$$
d(q, \mathscr{V}) \leq d\left(q^{\prime}, \mathscr{V}\right)+d\left(q, q^{\prime}\right) \leq 1-\chi(Q),
$$

and again we are done. So we may as well assume that $Q=Q_{0}$ is connected and, as we have seen, not an annulus.

Claim. The theorem holds if $Q$ is strongly $\partial$-compressible.

Proof of the Claim. Suppose there are disjoint $\partial$-compressing disks $F_{X} \subset X$ and $F_{Y} \subset Y$ for $Q$ in $M$. Let $Q_{x}$ and $Q_{y}$ denote the surfaces obtained from $Q$ by d-compressing $Q$ along $F_{X}$ and $F_{Y}$, respectively, and let $Q_{-}$denote the surface obtained by $\partial$-compressing along both disks simultaneously. (We use lowercase $x$ and $y$, to distinguish these from the surfaces $Q_{X}, Q_{Y}$ obtained by maximally compressing $Q$ into $X$ or $Y$, respectively.) A standard innermost disk, outermost arc argument between $F_{X}$ and a compressing disk for $Q$ in $X$ shows that $Q_{x}$ is compressible in $X$. Similarly, $Q_{y}$ is compressible in $Y$.

Each of $Q_{x}$ and $Q_{y}$ has at most two components, since $Q$ is connected. Suppose that $Q_{x}$ (say) is itself bicompressible. If it were strongly compressible, the same strong compression pair of disks would strongly compress $Q$, so we conclude that the inductive hypothesis applies to $Q_{x}$, and hence we apply the theorem to $Q_{x}$. One possibility is that one component of $Q_{x}$ is a tube-spanned recessed collar and the other (if there are two components) is $\partial$-parallel. But, by Lemma 4.6, this case implies that $Q$ is also a tube-spanned recessed collar, and we are done. The other possibility is that, for $q_{x}$ a component of the boundary of an essential component of $Q_{x}$,

$$
d\left(q_{x}, \mathscr{V}\right) \leq 1-\chi\left(Q_{x}\right)<1-\chi(Q) .
$$

This implies that

$$
d(q, \mathscr{V}) \leq d\left(q_{x}, \mathscr{V}\right)+d\left(q, q_{x}\right) \leq 1-\chi(Q),
$$

and again we are done. So we henceforth assume that $Q_{x}$ (respectively, $Q_{y}$ ) is compressible into $X$ (respectively, $Y$ ) but not into $Y$ (respectively, $X$ ).

It follows that $Q_{-}$is incompressible, for, if $Q_{-}$is compressible into $Y$, say, then such a compressing disk would be unaffected by the tunneling that recovers $Q_{x}$ from $Q_{-}$, and $Q_{x}$ would also compress into $Y$.

On the other hand, if $Q_{-}$is essential in $M$, then the claim follows from Proposition 2.5. In the proof of the claim, the only remaining case to consider is when $Q_{-}$ is incompressible and not essential, so all its components are $\partial$-parallel. Since $Q$ is connected, $Q_{-}$has at most three components. Suppose there are exactly three: 
$Q_{0}, Q_{1}, Q_{2}$. If the three are nested (that is, they can be arranged as $Q_{0}, Q_{1}, Q_{2}$ were in Lemma 4.5), then that lemma shows that the weakly incompressible $Q$ must be a tube-spanned recessed collar, as required. If no pairs of the three components of $Q_{-}$are nested, then $Q$ itself would be boundary-parallel, and so could not be compressible on the side towards $N$. Finally, suppose that two components ( $Q_{0}$ and $Q_{1}$, say) are nested, that $Q_{2}$ is $\partial$-parallel in their complement, and that $Q_{x}$, say, is obtained from $Q_{1}$ and $Q_{2}$ by tunneling between $Q_{1}$ and $Q_{2}$, so that $Q_{x}$ is $\partial$ parallel. $Q_{x}$ is also compressible; the compressing disk either also lies in a collar of $N$, or, via the parallelism to the boundary, the disk represents a compressing disk $D$ for $N$ in $M$ whose boundary is disjoint from $\partial Q_{x}$. In the latter case, for $q_{x}$ any component of $\partial Q_{x}$, we have $d\left(q_{x}, \partial D\right) \leq 1$. Then, for $q$ any component of $Q$,

$$
d(q, \partial D) \leq d\left(q_{x}, \partial D\right)+d\left(q, q_{x}\right) \leq 2 \leq 1-\chi(Q),
$$

and we are done. The former case can only arise if there are boundary components of $Q_{1}$ and $Q_{2}$ that cobound an annulus and that annulus is spanned by the tunnel. Moreover, since a resulting compressing disk for $Q_{x}$ lies in $N$ and so cannot persist into $Q$, the tunnel attaching $Q_{0}$ must be incident to that same boundary component of $Q_{1}$. It is easy then to see that $Q$ is a tube-spanned recessed product, where the two recessed surfaces are $Q_{0}$ and the union of $Q_{1}$ and $Q_{2}$ along their parallel boundary components.

Similar arguments apply if $Q_{-}$has one or two components. This completes the proof of the Claim.

Compressing a surface does not affect its boundary, so the theorem follows immediately from Lemma 5.5 and Proposition 2.5, unless the surface $Q_{X}$ - obtained by maximally compressing $Q$ into $X$ - has the property that each of its non-closed components is boundary-parallel in $M$. Of course, the symmetric statement holds also for the surface $Q_{Y}$ obtained by maximally compressing $Q$ into $Y$; indeed, all the ensuing arguments would apply symmetrically to $Q_{Y}$ simply by switching the labels $X$ and $Y$ throughout. So we henceforth assume that all components of $Q_{X}$ are either closed or $\partial$-parallel. There are some of the latter, since $Q$ has boundary.

Let $Q_{0}$ be an outermost $\partial$-parallel component of $Q_{X}$ that is not closed. That is, $Q_{0}$ is a component parallel to a subsurface of $\partial M$, and no component of $Q_{X}$ lies in the region of parallelism $R \cong Q_{0} \times I$. As in the proof of Lemma 5.5, we use the notation $X^{\prime} \subset X$ and $Y^{\prime} \supset Y$ for the two 3-manifolds into which $Q_{X}$ splits $M$, noting that, unlike for $Q$, some components of $Q_{X}$ may be closed. Note also the graph $\Gamma \subset Y^{\prime}$.

Case 1: Some such outermost region $R$ lies in $Y^{\prime}$. The other side of $Q_{0}$ lies in $X^{\prime}$, and so its interior is disjoint from $\Gamma$. Since $Q$ is connected, this implies that all of $Q$ lies in $R$. In particular, $\Gamma \subset R$, all compressing disks for $Q$ in $Y$ also lie in $R$, 
and $Q_{0}=Q_{X}$. Let $(D, \partial D) \subset(M, N)$ be a $\partial$-reducing disk for $M$, as in Lemma 5.5 , so that $\Gamma$ is disjoint from $D$, and $D \cap Q_{0}$ consists only of arcs that are essential in $Q_{0}$.

Any outermost such arc in $D$ cuts off a $\partial$-reducing disk $D_{0} \subset D$. Suppose first that $D_{0}$ lies in $M-R$, and let $Q_{0}^{\prime}$ be the surface created from $Q_{0}$ by $\partial$-compressing along $D_{0}$. By Lemma 2.3, $Q_{0}^{\prime}$ is incompressible, so all boundary components of $Q_{0}^{\prime}$ are essential in $\partial M$, unless $Q_{0}$ is an annulus that is parallel to $\partial M$ via $M-R$ as well. The latter would imply that $Q_{0}$ is a longitudinal annulus of a solid torus, and that $D$ is a meridian of that solid torus and we could have taken for $D_{0}$ the half of $D$ that does lie in $R$. In the general case, the union of $D_{0}$ with a disk of parallelism in $R$ gives a $\partial$-reducing disk for $M$ that is disjoint from $\partial Q_{0}^{\prime}$, so for any boundary component $q^{\prime}$ of $Q_{0}^{\prime}, d\left(q^{\prime}, \mathscr{V}\right) \leq 1$. Then, for $q$ any component of $\partial Q=\partial Q_{X}=\partial Q_{0}$

$$
d(q, \mathscr{V}) \leq d\left(q^{\prime}, \mathscr{V}\right)+d\left(q, q^{\prime}\right) \leq 2 \leq 1-\chi(Q),
$$

and we are done. In any case, we may as well then assume that $D_{0}$ lies in $R \subset Y^{\prime}$.

Since $\Gamma$ is disjoint from $D_{0}, D_{0}$ is a $\partial$-reducing disk for $Q$ as well, and lies in $Y$. A standard outermost-arc argument in $D_{0}$ shows that there is a compressing disk for $Q$ in $Y$ that is disjoint from $D_{0}$. Thus, $\partial$-reducing $Q$ along $D_{0}$ leaves a surface that is still bicompressible (since meridians of $\Gamma$ constitute compressing disks in $X$ ), but with $1-\chi(Q)$ reduced. The proof then follows by induction. (In fact, this argument can be enhanced to show directly that Case 1 simply cannot arise.)

It remains to consider the case in which all outermost components of $Q_{X}$ are $\partial$-parallel via a region that lies in $X^{\prime}$. We distinguish two further cases:

Case 2: There is nesting among the non-closed components of $Q_{X}$. We prove that $Q$ must be a tube-spanned recessed collar.

In this case, let $Q_{1}$ be a component that is not closed (so it is $\partial$-parallel), and is second-outermost; that is, the region of parallelism between $Q_{1}$ and $\partial M$ contains in its interior only outermost components of $Q_{X}$. Denote the union of the latter components by $Q_{0}$. The region between $Q_{0}$ and $Q_{1}$ is itself a product $R \cong Q_{1} \times I$, but one end contains $Q_{0}$ as a possibly disconnected subsurface. Since outermost components cut off regions lying in $X^{\prime}, R \subset Y^{\prime}$. We now argue much as in Case 1: Since $\Gamma \subset Y^{\prime}$ and $Q$ is connected, all of $\Gamma$ must lie in $R$, so $Q_{X}=Q_{1} \cup Q_{0}$. Let $(D, \partial D) \subset(M, N)$ be a $\partial$-reducing disk for $M$ as in Lemma 5.5, so that $D$ is disjoint from $\Gamma$ and intersects $Q_{X}$ only in arcs that are essential in $Q_{X}$. As in Case 1, each outermost arc of $D \cap Q_{X}$ in $D$ lies in $Q_{0}$.

Choose a complete collection of $\partial$-compressing disks $\mathscr{F}$ in the region of parallelism between $Q_{1}$ and $\partial M$, so that the complement $Q_{1}-\mathscr{F}$ is a single disk $D_{Q}$. Each disk in $\mathscr{F}$ is incident to $Q_{1}$ in a single arc. Now import the argument 
of Lemma 5.5 into this context: Let $E$ be a compressing disk for $Y$, here chosen so that $E \cap \mathscr{F}$ is minimized. This means, first of all, that $E \cap \mathscr{F}$ is a collection of arcs. As in the proof of Lemma 5.5, $\Gamma$ may be slid and isotoped so it is disjoint from $\mathscr{F}_{\text {. }} \Gamma$ is incident to $Q_{1}$, since $Q$ is connected. Since $D_{Q}$ is connected, the ends of $\Gamma$ on $D_{Q}$ may be slid within $D_{Q}$ so that ultimately $\Gamma$ is incident to $D_{Q}$ in a single point. The boundary $\partial E$ is necessarily incident to that end, since $Q$ is weakly incompressible. It follows that $\partial E$ cannot be incident to $Q$ only in $D_{Q}$ (or else $\partial E$ could be pushed off the end of $\Gamma$ in $D_{Q}$ ), so $\partial E$ must intersect the arcs $\partial \mathscr{F} \cap Q_{1}$. Let $\beta \subset \mathscr{F} \cap E$ be outermost in $E$ among all arcs incident to components of $\partial \mathscr{F} \cap Q_{1}$. Let $E_{0}$ be the disk that $\beta$ cuts off from $E$.

If both ends of $\beta$ were in $\mathscr{F} \cap Q_{1}$, then, since each disk of $\mathscr{F}$ is incident to $Q_{1}$ in a single arc, $\beta$ would cut off a subdisk of $\mathscr{F}$ that could be used to alter $E$, creating a compressing disk for $Y$ that intersects $\mathscr{F}$ in fewer points. We conclude that the other end of $\beta$ is on $Q_{0}$. Since $\beta$ is outermost among those arcs of $E \cap \mathscr{F}$ incident to $D_{Q}, \partial E_{0}$ traverses the end of $\Gamma$ on $D_{Q}$ exactly once. So, as in the proof of Lemma 5.5, it can be used to slide and isotope an edge $\rho$ of $\Gamma$ until it coincides with $\beta$. Hence, the edge $\rho \subset \Gamma$ can be made into a vertical arc (that is, an $I$-fiber) in the product structure $R=Q_{1} \times I$.

Using that product structure and an essential circle in the component of $Q_{0}$ that is incident to $\rho$, the arc $\rho$ can be viewed as part of a vertical incompressible annulus $A$ with ends on $Q_{1}$ and $Q_{0}$. Now apply the argument of Lemma 5.5 again: $A-\rho$ is a disk $E^{\prime}$. Since $E^{\prime}$ is a disk, we use the argument of Lemma 5.5 to slide and isotope the edges of $\Gamma-\rho$ until they are disjoint from $E^{\prime}$. After these slides, $E^{\prime}$ is revealed as a compressing disk for $Q$ in $Y$. On the other hand, if there is in fact any edge $\gamma$ in $\Gamma-\rho$, the compressing disk for $Q$ in $X$ given by the meridian of $\eta(\gamma)$ would be disjoint from $E^{\prime}$, contradicting the weak incompressibility of $Q$. So we conclude that in fact $\Gamma=\rho$, and so, other than the components of $Q_{X}$ incident to the ends of $\rho$, each component of $Q_{X}$ is a component of $Q$; since $Q$ is connected, there are no such other components. That is, $Q$ is obtained by tubing $Q_{1}$ to the connected $Q_{0}$ along $\rho$, and so is a tube-spanned recessed collar. This completes the argument in this case.

Case 3: All non-closed components of $Q_{X}$ are outermost among the components of $Q_{X}$. We show that $Q$ is strongly $\partial$-compressible; the proof then follows from the previous Claim.

We have already seen that all non-closed components of $Q_{X}$ are $\partial$-parallel through $X^{\prime}$. Choose a $\partial$-reducing disk $(D, \partial D) \subset(M, N)$ for $M$ as in Lemma 5.5, so that $D$ is disjoint from the graph $\Gamma$, intersects $Q_{X}$ minimally, and intersects $Q$ only in arcs that are essential in $Q_{X}$. Although there is no nesting among the components of $Q_{X}$, it is not immediately clear that the $\operatorname{arcs} D \cap Q_{X}$ are not nested in $D$. However, it is true that each outermost arc cuts off a subdisk of $D$ that lies in 
$X^{\prime}$, as shown above in the proof of Case 1 . In what follows, $D^{\prime}$ will represent either $D$, if no $\operatorname{arcs}$ of $D \cap Q_{X}$ are nested in $D$, or a disk cut off by a second-outermost arc of intersection $\lambda_{0}$, if there is nesting. Let $\Lambda \subset D^{\prime}$ denote the collection of arcs $D^{\prime} \cap Q$; one of these arcs (namely, $\lambda_{0}$ ) may be on $\partial D^{\prime}$.

Consider how a compressing disk $E$ for $Q$ in $Y$ intersects $D^{\prime}$. All closed curves in $D^{\prime} \cap E$ can be removed by a standard innermost-disk argument redefining $E$. Any arc in $D^{\prime} \cap E$ must have its ends on $\Lambda$; a standard outermost-arc argument can be used to remove any that has both ends on the same component of $\Lambda$. If any component of $\Lambda-\lambda_{0}$ is disjoint from all the arcs $D^{\prime} \cap E$, then $Q$ could be $\partial$-compressed without affecting $E$. This reduces $1-\chi(Q)$ without affecting bicompressibility, so we would be done, by induction. Hence, we restrict to the case in which each arc-component of $\Lambda-\lambda_{0}$ is incident to some arc-components of $D^{\prime} \cap E$. See figure.

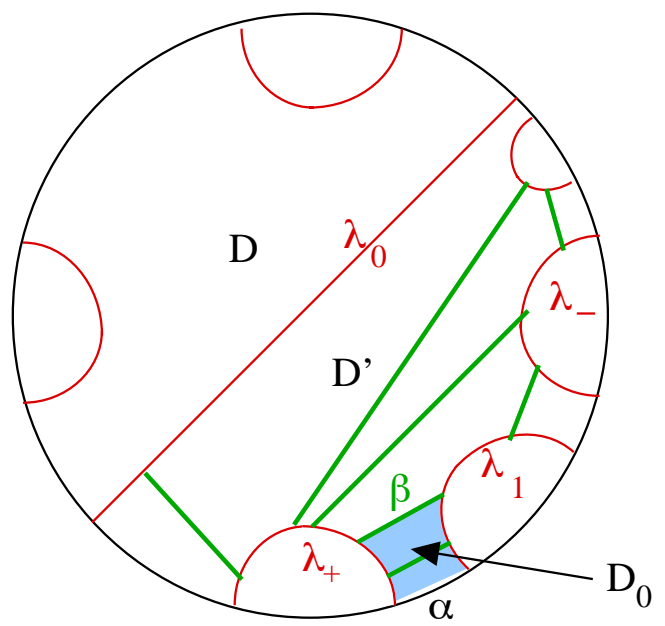

It follows that there is at least one component $\lambda_{1} \neq \lambda_{0}$ of $\Lambda$ with this property: any arc of $D^{\prime} \cap E$ that has one end incident to $\lambda_{1}$ has its other end incident to one of the (at most two) neighboring components $\lambda_{ \pm}$of $\Lambda$ along $\partial D^{\prime}$. (Possibly, one or both of $\lambda_{ \pm}$are $\lambda_{0}$.) Let $\beta$ be the arc in $E$ outermost among all arcs of $D^{\prime} \cap E$ that are incident to the special arc $\lambda_{1}$. We then know that the other end of $\beta$ is incident to (say) $\lll_{+}$, and that the disk $E_{0} \subset E$ cut off by $\beta$ from $E$, although possibly incident to $D$ in its interior, contains no arc of intersection with $D$ that is incident to $\lll_{1}$.

Let $D_{0}$ be the rectangle in $D$ whose sides consist of subarcs of $\lambda_{1}, \lambda_{+}, \partial D$, and all of $\beta$. Although $E$ may intersect this rectangle, our choice of $\beta$ as outermost among arcs of $D \cap E$ incident to $\lambda_{1}$ guarantees that $E_{0}$ is disjoint from the interior of $D_{0}$, and so is incident to it only in the $\operatorname{arc} \beta$. The union of $E_{0}$ and $D_{0}$ along $\beta$ is a disk $D_{1} \subset Y$ whose boundary consists of the $\operatorname{arc} \alpha=\partial M \cap \partial D_{0}$ and an arc 
$\beta^{\prime} \subset Q$. The latter arc is the union of the two $\operatorname{arcs} D_{0} \cap Q$ and the $\operatorname{arc} E_{0} \cap Q$. If $\beta^{\prime}$ is essential in $Q$, then $D_{1}$ is a $\partial$-compressing disk for $Q$ in $Y$ that is disjoint from the boundary-compressing disk in $X$ cut off by $\lambda_{1}$. So, if $\beta^{\prime}$ is essential, then $Q$ is strongly $\partial$-compressible, and we are done by the Claim.

Suppose finally that $\beta^{\prime}$ is inessential in $Q$. Then $\beta^{\prime}$ is parallel to an arc on $\partial Q$ and so, via this parallelism, the disk $D_{1}$ is itself parallel to a disk $D_{2}$ that is disjoint from $Q$ and either is $\partial$-parallel in $M$ or is itself a $\partial$-reducing disk for $M$. If $D_{2}$ is a $\partial$-reducing disk for $M$, then $\partial D_{2} \in \mathscr{V}, d(2, \mathscr{V}) \leq 1$, and we are done. On the other hand, if $D_{2}$ is parallel to a subdisk of $\partial M$, then an outermost arc of $\partial D$ in that disk (possibly the arc $\alpha$ itself) can be removed by an isotopy of $\partial D$, lowering $|D \cap Q|=\left|D \cap Q_{X}\right|$. This contradicts our original choice of $D$.

\title{
References
}

[Bachman and Schleimer 2005] D. Bachman and S. Schleimer, "Distance and bridge position", Pacific J. Math. 219:2 (2005), 221-235. MR MR2175113 Zbl 1086.57011

[Casson and Gordon 1987] A. J. Casson and C. M. Gordon, "Reducing Heegaard splittings", Topology Appl. 27:3 (1987), 275-283. MR 89c:57020 Zbl 0632.57010

[Hartshorn 2002] K. Hartshorn, "Heegaard splittings of Haken manifolds have bounded distance", Pacific J. Math. 204:1 (2002), 61-75. MR 2003a:57037 Zbl 1065.57021

[Hempel 2001] J. Hempel, "3-manifolds as viewed from the curve complex", Topology 40:3 (2001), 631-657. MR 2002f:57044 Zbl 0985.57014

[Jones and Scharlemann 2001] M. Jones and M. Scharlemann, "How a strongly irreducible Heegaard splitting intersects a handlebody”, Topology Appl. 110:3 (2001), 289-301. MR 2001m:57033 Zbl 0974.57011

[Rubinstein and Scharlemann 1996] H. Rubinstein and M. Scharlemann, "Comparing Heegaard splittings of non-Haken 3-manifolds", Topology 35:4 (1996), 1005-1026. MR 97j:57021 Zbl 0858. 57020

[Scharlemann 1998] M. Scharlemann, "Local detection of strongly irreducible Heegaard splittings", Topology Appl. 90:1-3 (1998), 135-147. MR 99h:57040 Zbl 0926.57018

[Scharlemann 2002] M. Scharlemann, "Heegaard splittings of compact 3-manifolds", pp. 921-953 in Handbook of geometric topology, edited by R. Daverman and R. Sher, North-Holland, Amsterdam, 2002. MR 2002m:57027 Zbl 0985.57005

[Scharlemann and Thompson 1994] M. Scharlemann and A. Thompson, "Thin position and Heegaard splittings of the 3-sphere", J. Differential Geom. 39:2 (1994), 343-357. MR 95a:57026 Zbl 0820.57005

Received March 28, 2005.

\author{
MARTIN SCHARLEMANN \\ MATHEMATICS DEPARTMENT \\ UNIVERSITY OF CALIFORNIA AT SANTA BARBARA \\ SANTA BARBARA, CA 93106 \\ UNITED STATES \\ mgscharl@math.ucsb.edu
}

